\title{
Compatibility and Mechanical Properties of Binary Blends Composed of Aromatic Poly(ether sulfone) and Poly $(m-$ phenyleneisophthalamide) by Solution Blending
}

\author{
Hideo Hayashi, Masa-aki KaKimoto, ${ }^{*}$ and Yoshio Imai, ${ }^{*}$ \\ Polymer Research Laboratory, Idemitsu Petrochemical Co., Ltd., \\ Anegasaki-kaigan, Ichihara, Chiba 299-01, Japan \\ * Department of Organic and Polymeric Materials, Tokyo Institute of Technology, \\ Meguro-ku, Tokyo 152, Japan
}

(Received July 20, 1993)

\begin{abstract}
Poly(oxy-1,4-phenylenesulfonyl-1,4-phenylene) (PES) and poly( $m$-phenyleneisophthalamide) (aramid) were blended by a conventional solution blending method. The PES/aramid solution blended films, prepared by casting from the $N, N$-dimethylacetamide (DMAc) solution of the two polymer mixtures, exhibited two glass transition temperatures and gave phase-separated translucent or opaque films over the entire PES/aramid composition ranges. The morphology observed by scanning electron microscope also revealed that the blend films had two phase structure with the PES domain dispersed in the aramid matrix. The tensile modulus and tensile strength of the films were somewhat low because of the heterogeneous morphology compared with the component polymer films, while the elongation at break of the blend films increased remarkably, reaching to about $190 \%$, indicative of the tough and ductile films. The annealing of the blend films afforded high temperature resistant films with high tensile modulus and strength due to both crystallization and partial cross-linking of the aramid component.

KEY WORDS Solution Blending / Poly(ether sulfone)-Aramid Blend /

Compatibility / Mechanical Properties / Crystallization Behavior /
\end{abstract}

Aromatic poly(ether sulfone)s such as poly(oxy-1,4-phenyleneisopropylidene-1,4-phenyleneoxy-1,4-pheylenesulfonyl-1,4-phenylene) $(\mathrm{PSF})^{1}$ and poly(oxy-1,4-phenylenesulfonyl1,4-phenylene) (PES) ${ }^{2}$ are well known as highperformance engineering thermoplastics. However it is quite difficult that one kind of poly(ether sulfone) possesses all the characteristics and functions demanded. Polymer blends exhibit a better balance of properties than each of the polymers alone. Hence, in recent years, a number of novel polymer blends based on poly(ether sulfone)s have been developed. For example, PSF-poly( $p$-phenylene sulfide) (PPS), ${ }^{3-7}$ PSF-poly(ether ketone) (PEK), ${ }^{8,9}$ PES-PPS,${ }^{10}$ and PES-PEK ${ }^{11-13}$ have been reported.

We recently reported that the binary blend of PES and aramid 44I, poly[ $N, N^{\prime}$-(oxydi-pphenylene)isophthalamide], obtained through solution blending at the PES/aramid 30/70 composition on a weight basis gave a transparent compatible blend film despite being thermodynamically immiscible. ${ }^{14}$ Further, we found that the blend film had high elongation at break $(c a .80 \%)$ at the PES/aramid 50/50 composition, ${ }^{15}$ and that the blend film showed an interesting crystallization behavior at the PES/aramid 30/70 composition. Since PES is an amorphous polymer and the aramid is a dormant crystalline, the crystallization of the aramid was induced and accelerated dra-

\footnotetext{
† To whom correspondence should be addressed.
} 
matically at $300^{\circ} \mathrm{C}$ by blending with PES, converting into a high temperature resistant blend film. ${ }^{16,17}$

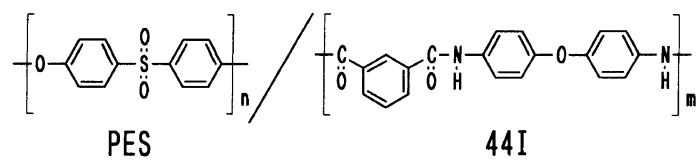

In this work, we employed poly ( $m$-phenyleneisophthalamide) (PMIA), a common aramid having no ether linkage and more rigid structure than aramid 44I, as the aramid component for PES/aramid blend system. We herein report compatibility, mechanical properties, and crystallization behavior of PES/PMIA blend by a conventional solution blending.

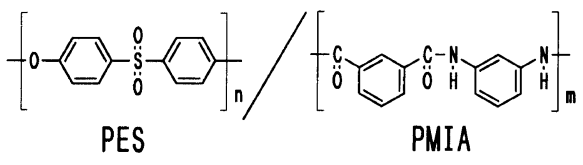

\section{EXPERIMENTAL}

\section{Materials}

Aromatic poly(ether sulfone) PES was supplied by Imperial Chemical Industries, Ltd., as Victrex PES 4800P, and used as received. The inherent viscositiy of PES in $\mathrm{N}$-methyl-2pyrrolidone(NMP) was $0.5 \mathrm{dlg}^{-1}$, measured at a concentration of $0.5 \mathrm{~g} \mathrm{dl}^{-1}$ at $30^{\circ} \mathrm{C}$. All the solvents such as $N, N$-dimethylacetamide (DMAc) and NMP were purified by distillation under reduced pressure.

\section{Preparation of Aramid PMIA}

Aramid PMIA was prepared by a coventional low temperature solution polycondensation method, ${ }^{18}$ starting from isophthaloyl chloride and $m$-phenylenediamine. Isophthaloyl chloride and $m$-phenylenediamine were obtained commercially. and purified by vacuum distillation. A typical procedure for the synthesis of aramid PMIA is as follows. In a $200 \mathrm{ml}$ roundbottom flask equipped with a mechanical stirrer were placed $m$-phenylenediamine $(5.40 \mathrm{~g}, 0.05$ mol) and NMP $(100 \mathrm{ml})$. The solution was chilled with an ice-water bath. To the solution was added solid isophthaloyl chloride $(10.2 \mathrm{~g}$, $0.05 \mathrm{~mol}$ ) and the solution was stirred at $0^{\circ} \mathrm{C}$ for $3 \mathrm{~h}$ under nitrogen. The viscous solution thus obtained was poured into methanol. The precipitated polymer was collected, washed with refluxing methanol, and dried under vacuum. The inherent viscosity was $1.4 \mathrm{dl} \mathrm{g}^{-1}$, measured at a concentration of $0.5 \mathrm{~g} \mathrm{dl}^{-1}$ in NMP at $30^{\circ} \mathrm{C}$.

\section{Preparation of Solution Blended Films}

A typical example of the preparation of the solution blended film of the PES/aramid 50/50 composition on a weight basis is as follows. In a flask equipped with a mechanical stirrer, a nitrogen inlet, and a condenser with a drying tube, was placed $10 \mathrm{ml}$ of DMAc. This was cooled with an ice-ethanol bath to $-10^{\circ} \mathrm{C}$. To the solvent was added $1.0 \mathrm{~g}$ of the aramid. The mixture was stirred at $-10^{\circ} \mathrm{C}$ for $1 \mathrm{~h}$ and then the temperature was raised slowly to room temperature under nitrogen. To the solution was added $1.0 \mathrm{~g}$ of PES in one portion, and the mixture was stirred at room temperature for $24 \mathrm{~h}$. The solution was then cast on a glass plate, followed by drying successively under vacuum at $0-5^{\circ} \mathrm{C}$ for $6 \mathrm{~h}$, at room temperature for $24 \mathrm{~h}$, at $100^{\circ} \mathrm{C}$ for $24 \mathrm{~h}$, and at $200^{\circ} \mathrm{C}$ for 7 days, and washing with methanol for $24 \mathrm{~h}$. The film was further dried under vacuum at $200^{\circ} \mathrm{C}$ for 2 days. The solution blended films of about $30 \mu \mathrm{m}$ were translucent or opaque in appearance, depending on the PES/aramid composition.

\section{Annealing of Samples}

Annealing of the blend film was carried out under vacuum at $330^{\circ} \mathrm{C}$ for $12 \mathrm{~h}$.

\section{Measurements}

Differential scanning calorimetry (DSC) was performed with a Shimadzu thermal analyzer DSC-41M at a heating rate of $20^{\circ} \mathrm{Cmin}^{-1}$ under nitrogen atmosphere. The midpoint of the slope change of the heat capacity plot of 
the DSC first scan was taken as glass transition temperature $\left(T_{\mathrm{g}}\right)$. Tensile properties were measured at room temperature using a Toyo Baldwin Tension UTM-II-20 with film specimens of $5 \mathrm{~mm}$ wide, $40 \mathrm{~mm}$ gauge length, and $30 \mu \mathrm{m}$ thick. Five specimens for each sample were measured at a strain rate of $20 \% \mathrm{~min}^{-1}$. The sample having the maximum elongation at break was taken as the data for elongation at break and tensile strength. The scanning electron micrographs were obtained with a JEOL JSM-25SIII scanning electron microscope (SEM) at a accelerating voltage of $15-30 \mathrm{kV}$. Sample preparation for the observation of morphology with SEM was carried out as follows: the blend film was fractured at liquid nitrogen temperature, and the fracture surface was treated with chloroform at room temperature for $24 \mathrm{~h}$ to remove the PES domain. After that, the surface was coated with gold. Wide angle X-ray diffraction profiles were obtained for the film specimens on a Rigakudenki XG X-ray diffraction apparatus using nickel-filtered $\mathrm{Cu}-K_{\alpha}$ radiation $(35 \mathrm{kV}$, $20 \mathrm{~mA}$ ).

\section{RESULTS AND DISCUSSION}

\section{Compatibility of Binary Polymer Blends}

The compatibility between aramid PMIA and PES was first studied for the ternary solution blends in DMAc with the composition of $30 / 70,40 / 60,50 / 50,60 / 40$, and $80 / 20$ for PES/PMIA, and of $20 / 80$ for polymer/solvent on a weight basis. All the PES/PMIA solutions were visually heterogeneous, that is, incompatible. Based on the opaque level, the compatibility of the PES/PMIA solutions appeared to decrease with the increase of the relative PES concentrations, except for PES $80 \mathrm{wt} \%$.

The ternary heterogeneous solutions were cast onto glass plates giving binary polymer blend films. Naturally all the as-prepared films were opaque or translucent. These films were subjected to thermal characterization by using DSC. Figure 1 shows the DSC curves of the

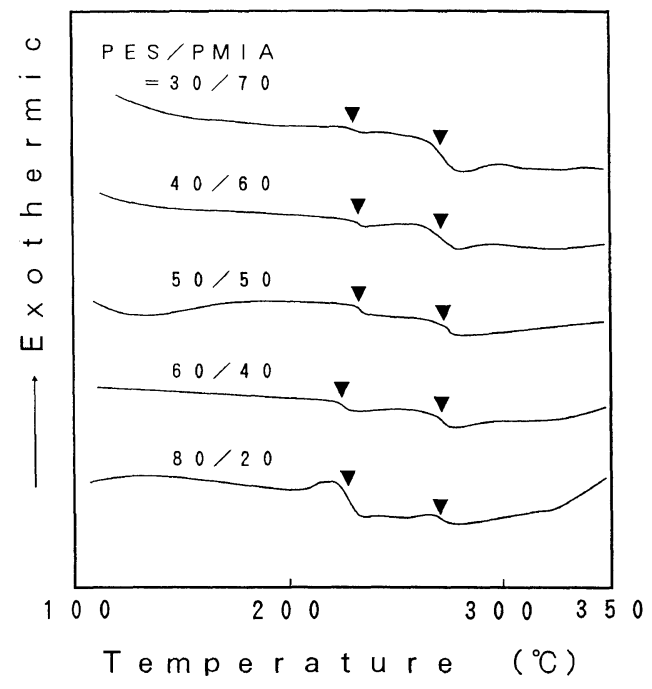

Figure 1. DSC thermograms of the first runs for the blend films.

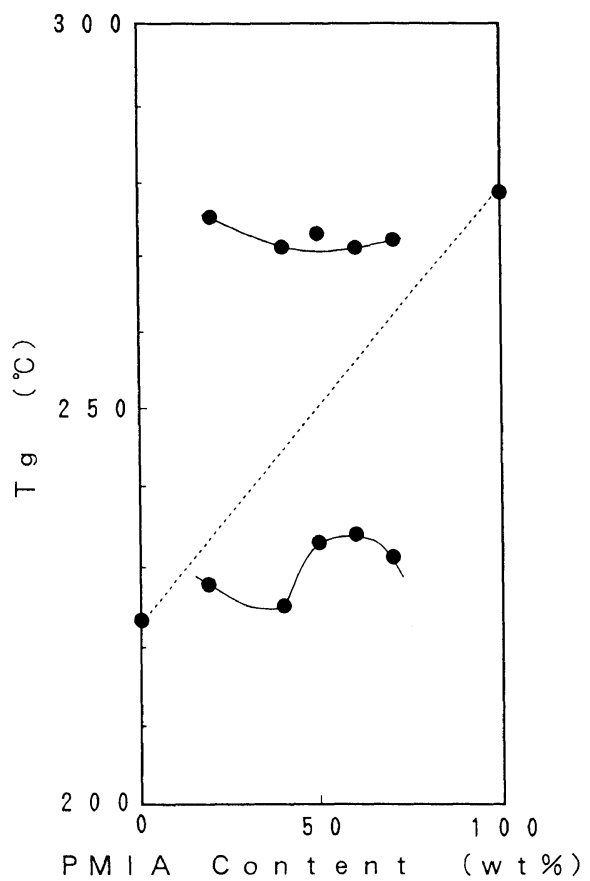

Figure 2. Composition dependence of $T_{\mathrm{g}}$ for the blend films. A dashed line is that calculated $T_{\mathrm{g}}$ values based on linear mixture rule.

first runs of the solution blended films over the entire composition ranges. All the films exhibited apparently two $T_{\mathrm{g}}$ 's corresponding to 

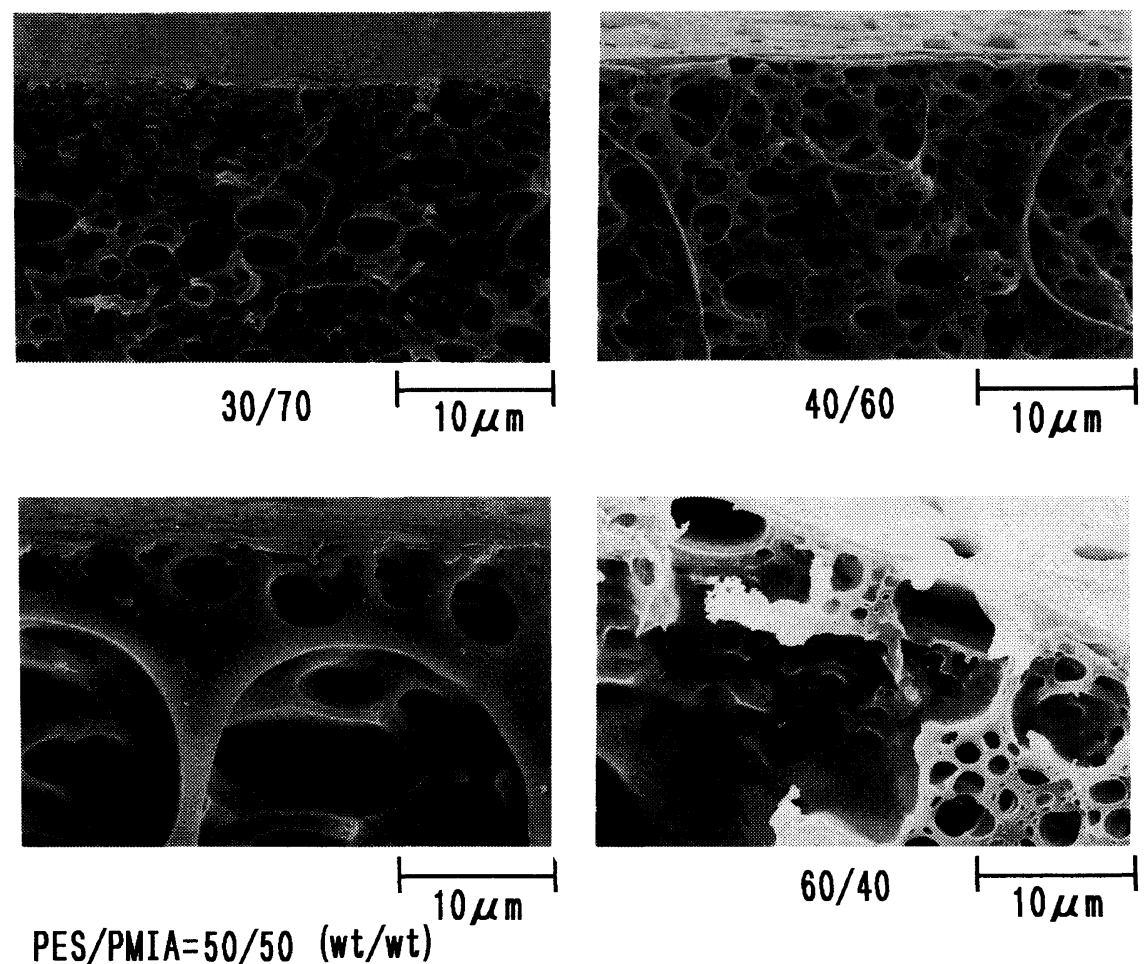

Figure 3. SEM micrographs of fractured surface of the blend films.

phase separated blends giving opaque or translucent films. Figure 2 shows the composition dependence of $T_{\mathrm{g}}$ for the solution blended films. These films displayed two $T_{\mathrm{g}}$ 's corresponding to the composition of each phase. Although the $T_{\mathrm{g}}$ 's for the PES/PMIA 30/70, $40 / 60$, and 50/50 compositions lay inner shift from the individual constituent polymers $\left(225^{\circ} \mathrm{C}\right.$ for PES and $280^{\circ} \mathrm{C}$ for PMIA), the lower $T_{\mathrm{g}}$ of PES rich phase for the PES/PMIA $60 / 40$ composition is very close to the $T_{\mathrm{g}}$ of pure PES, and the film had large phase separated structure visually.

Figure 3 shows the SEM micrographs of the solution blended films. All the films were heterogeneous and had two phase structure with the PES domain dispersed in the PMIA matrix. The diameter of the PES domain became gradually larger with the increase of the PES composition.

\section{Mechanical Properties of Solution Blended Films}

Figure 4 shows the stress-strain curves for the films of PES, PMIA, and the PES/PMIA 50/50 blend. A comparison of the curves showed a significant increase in elongation at break for the solution blended film. The composition dependences of tensile modulus, tensile strength at yield and elongation at break for the solution blended films are shown in Figures 5, 6, and 7, respectively. The tensile modulus and tensile strength of these films exhibited negative deviation from a simple addition rule of mixture. Figure 8 shows the morphology of the deformed tensile specimens for the PES/PMIA 30/70, 40/60, 50/50, and $60 / 40$ blends, observed in the fractured surface of the specimen without treating with chloroform. The dispersed PES particles appeared for the PES/PMIA 50/50 and 60/40 films, either separated from or loosely attached to the 


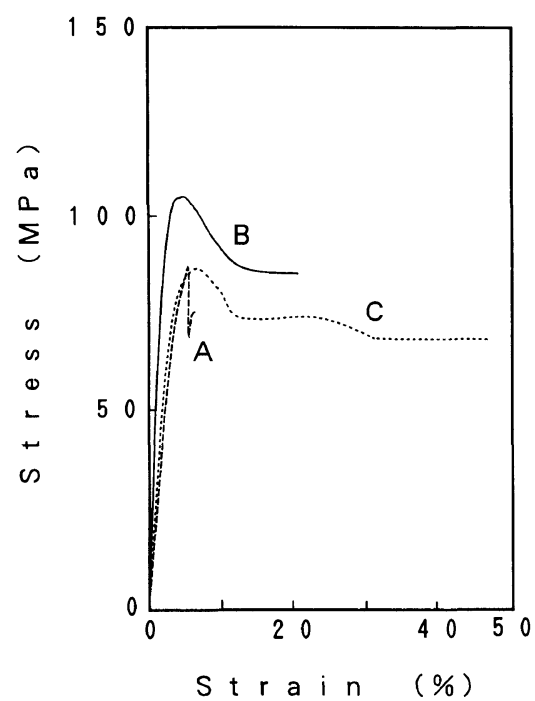

Figure 4. Stress-strain curves for the films: (A) PES; (B) PMIA; (C) PES/PMIA 50/50 blend film.

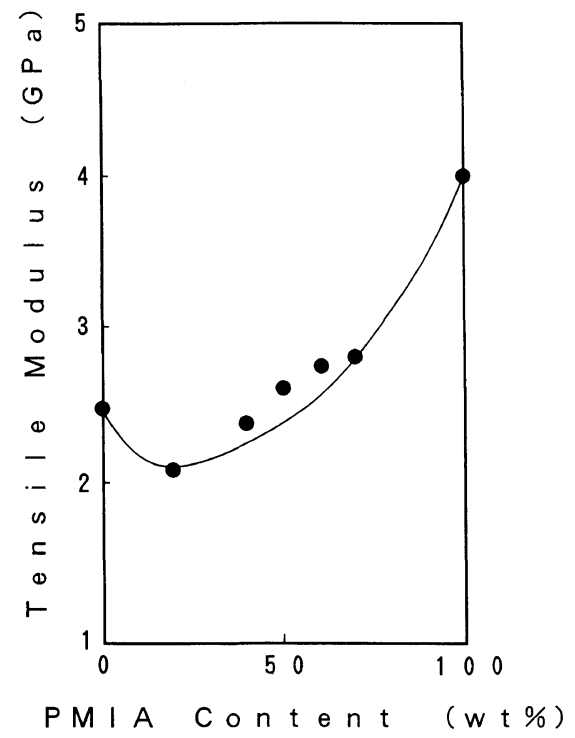

Figure 5. Composition dependence of tensile modulus for the blend films.

PMIA matrix. The lack of interfacial adhesion was evidenced by the absence of adhered PMIA on the surface of PES particles and by the large number of craters produced by pulled-out particles during the tensile testing or fracture of the specimens. From the observation, the

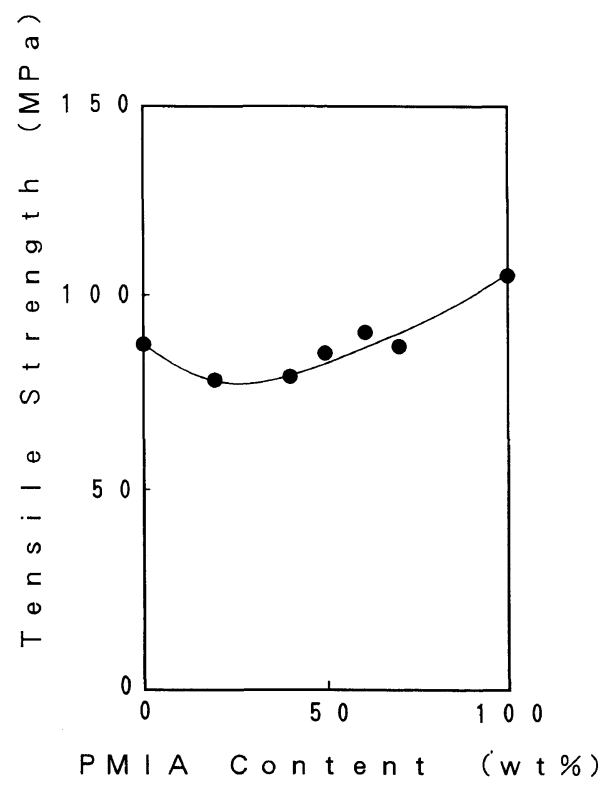

Figure 6. Composition dependence of tensile strength for the blend films.

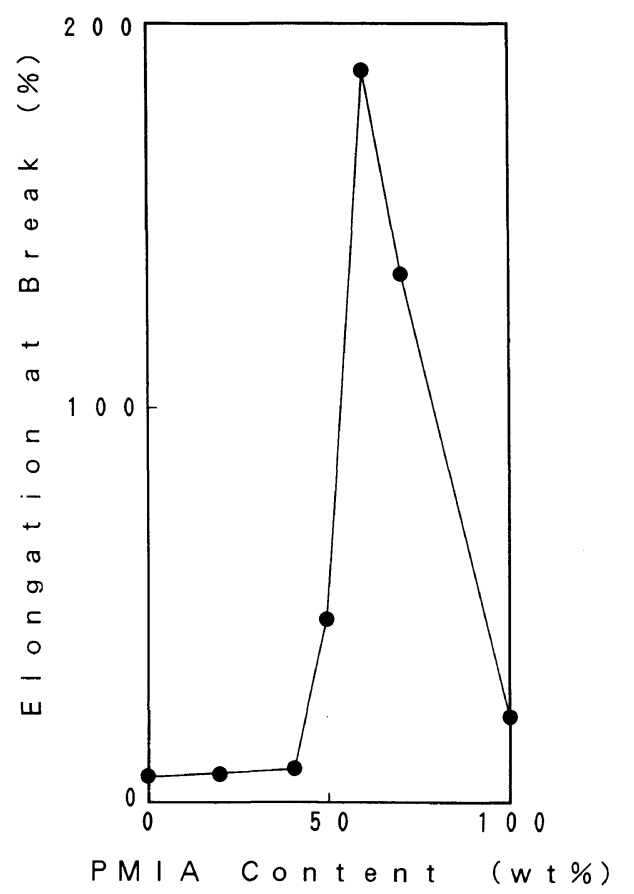

Figure 7. Composition dependence of elongation at break for the blend films. 

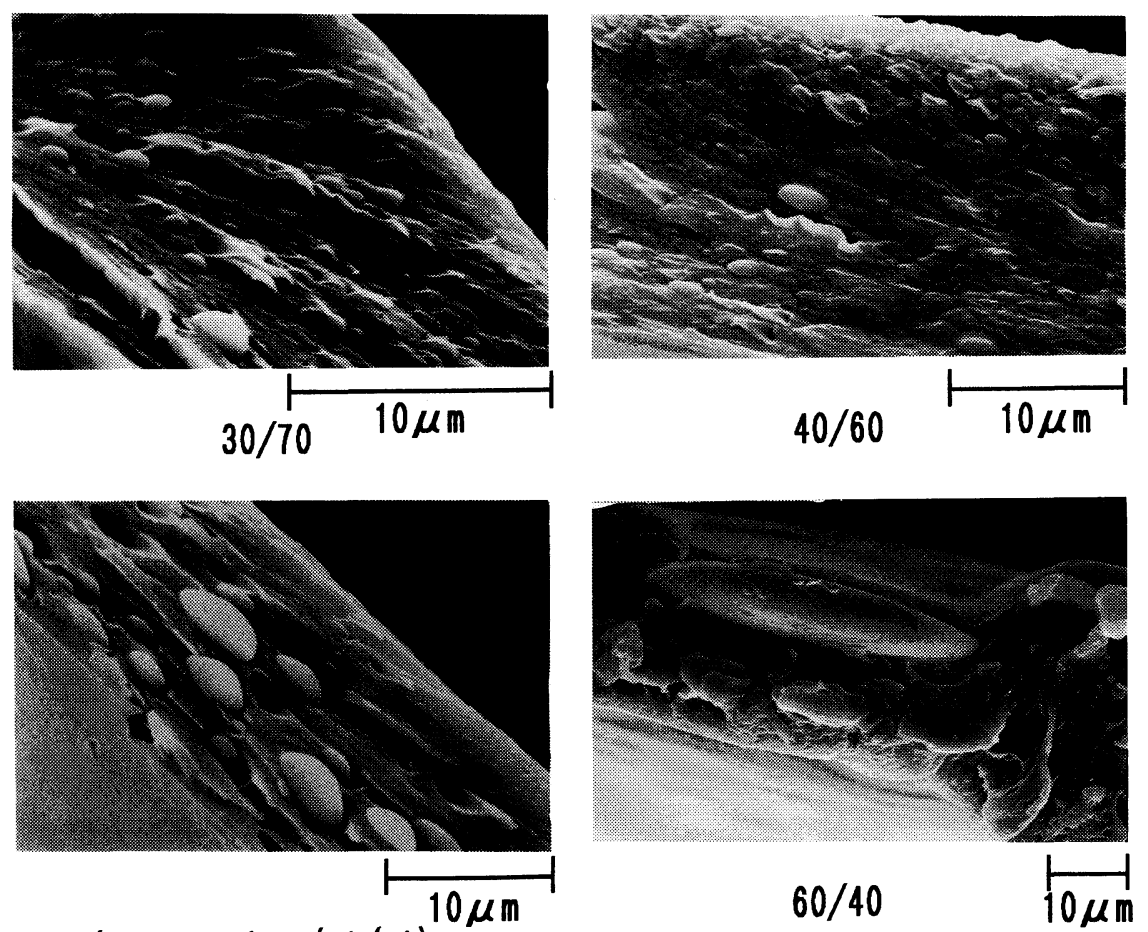

PES/PMIA $=50 / 50 \quad(w t / w t)$

Figure 8. SEM micrographs of fractured surface of the deformed tensile specimen without etching out PES by chloroform.

low elongation at break for these films are attributed to no interphase adhesion. On the other hand, the solution blended films with the PES/PMIA 30/70 and 40/60 compositions had a characteristic of high elongation at break over $100 \%$. The morphology of the deformation phase for the PES/PMIA 30/70 and 40/60 blends showed interphase adhesion between the PES particles and the PMIA matrix (Figure 8), which resulted in ductile films.

\section{Annealing of Solution Blended Films}

We recently reported that the crystallization of aramid 44I in the PES/44I solution blended film with the $30 / 70$ composition was accelerated dramatically by the presence of PES during annealing of the film, and the annealed film exhibited high modulus (350 MPa) at high temperature $\left(400^{\circ} \mathrm{C}\right){ }^{14-17}$ The acceleratedcrystallization was ascribed to the increase of chain mobility of 44I by blending with PES, probably caused by the decrease of $T_{\mathrm{g}}$ by blending with low $T_{\mathrm{g}}$ component (PES). In addition, the other contributions are due to the partial dissociation of 44I-44I interactions (hydrogen bonded $\mathrm{N}-\mathrm{H}$ ) and the "up-hill diffusion" 16 associated with the liquid-liquid phase separation.

The effect as described above may be expected also for the present PES/PMIA system. That is, the crystallization of PMIA in the blend films would be accelerated remarkably during annealing, resulting in high temperature resistant films. The solution blended films were subjected to annealing at $330^{\circ} \mathrm{C}$ (above the $T_{\mathrm{g}}$ of PMIA) for $12 \mathrm{~h}$ in order to crystallize PMIA. Figure 9 shows the temperature dependences of dynamic storage modulus and loss modulus for the nonannealed and annealed films of both neat 
PMIA and the solution blends with the PES/PMIA 50/50 composition. It is apparent that the $T_{\mathrm{g}}$ of PMIA shifted to higher temperature by about $50-80^{\circ} \mathrm{C}$ in both the solution blended film and the neat PMIA film by annealing, and these annealed films exhibited high modulus $(>1 \mathrm{GPa})$ at high temperature $\left(300^{\circ} \mathrm{C}\right)$ because of their high $T_{\mathrm{g}}$. The shift of the $T_{\mathrm{g}}$ toward higher temperature is probably due to the crystallization or partial cross-linking reaction of PMIA. Figure 10

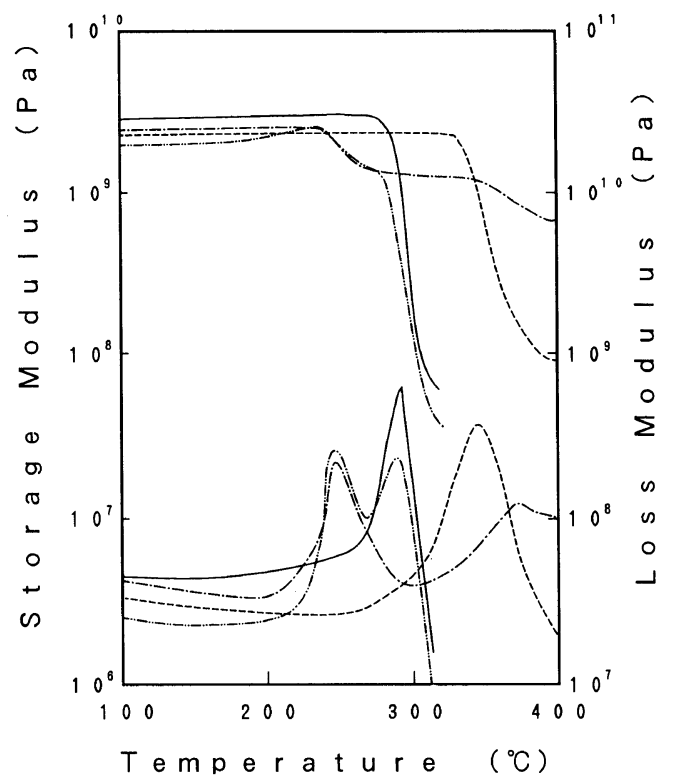

Figure 9. Temperature dependences of dynamic storage modulus and loss modulus for non-annealed neat PMIA film (- - , annealed neat PMIA film (-----), non-annealed PES/PMIA 50/50 blend film (- - - ) , and annealed PES/ PMIA 50/50 blend film (-- ). shows the X-ray diffraction patterns for the annealed neat PMIA and the annealed solution blended film with the PES/PMIA 50/50

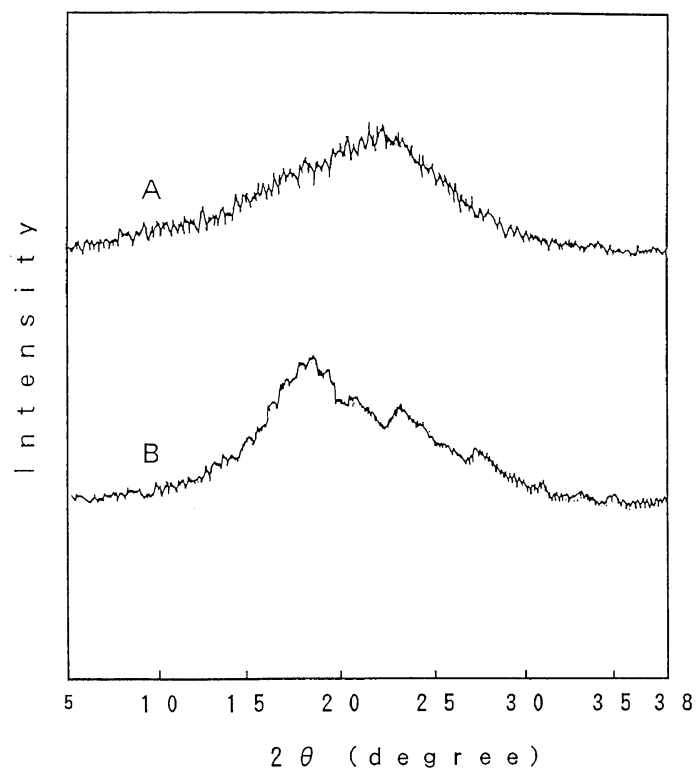

Figure 10. X-Ray diffraction patterns for annealed films: (A) annealed PMIA film; (B) annealed PES/PMIA 50/50 blend film.

Table I. Solubility of non-annealed and annealed films ${ }^{\mathrm{a}}$

\begin{tabular}{lccc}
\hline & PES & $\begin{array}{c}\text { Solution } \\
\text { blend } \\
50 / 50\end{array}$ & PMIA \\
& & $50 / 5$ & + \\
Non-annealed film & + & + & + \\
Annealed film & + & - & - \\
\hline
\end{tabular}

a In concentrated sulfuric acid: +, soluble; - , insoluble.

Table II. Mechanical properties of the annealed films

\begin{tabular}{ccccc}
\hline $\begin{array}{c}\text { Composition PES/PMIA } \\
(\mathrm{wt} / \mathrm{wt})\end{array}$ & Film $^{\mathrm{a}}$ & $\begin{array}{c}\text { Tensile strength } \\
(\mathrm{MPa})\end{array}$ & $\begin{array}{c}\text { Elongation at break } \\
(\%)\end{array}$ & $\begin{array}{c}\text { Tensile modulus } \\
(\mathrm{GPa})\end{array}$ \\
\hline \multirow{2}{*}{$0 / 100$} & Non-Ann. & 105 & 22 & 4.0 \\
& Ann. & 161 & 9.2 & 4.7 \\
$30 / 70$ & Non-Ann. & 86 & 134 & 2.8 \\
& Ann. & 123 & 4.0 & 4.1 \\
$50 / 50$ & Non-Ann. & 86 & 46 & 2.6 \\
& Ann. & 120 & 6.2 & 3.5 \\
\hline
\end{tabular}

a Non-Ann., non-annealed film; Ann., film annealed at $330^{\circ} \mathrm{C}$ for $12 \mathrm{~h}$. 
composition. It is evident that the annealed blend film was semicrystalline, while the annealed neat PMIA film was amorphous. Table I shows the results of solubility test in concentrated sulfuric acid. Both the annealed neat PMIA and the annealed blend film were quite insoluble in the solvent. From these results, it was concluded that the reason for the shift of $T_{\mathrm{g}}$ toward higher temperature was both crystallization and partial cross-linking reaction through annealing for the blend film, whereas that for the neat PMIA film was just cross-linking reaction.

\section{Mechanical Properties of Annealed Solution Blended Films}

The tensile properties of the annealed blend films are summerized in Table II. It is noteworthy that the tensile strength and modulus of both the neat PMIA and the blend films could be improved greatly by annealing, while the elongation at break drastically lowered. The increases in the tensile strength and modulus are attributed to the partially crystalline and/or cross-linked structures of the aramid component.

\section{CONCLUSION}

The solution blending of PES and aramid PMIA in DMAc afforded heterogeneous blend solutions, which in turn gave translucent or opaque polymer blend films. The morphology of the blend films exhibited two phase structure with the PES domain dispersed in the PMIA matrix. The blend film with the PES/PMIA $40 / 60$ composition had a characteristic of high elongation at break (about 190\%). The annealing of the blend films afforded high temperature resistant films with high tensile modulus and high tensile strength due to partially crystalline and cross-linked structures. Therefore, the PES/PMIA solution blended films are considered to be promising high temperature plastic films.

\section{REFERENCES}

1. R. N. Johnson, A. G. Farnham, R. A. Clendinning, W. F. Hale, and C. N. Merriam, J. Polym. Sci., A-1, 5, 2375 (1967).

2. T. E. Attwood, D. A. Barr, T. King, A. B. Newton, and J. B. Rose, Polymer, 18, 359 (1977).

3. H. Zeng and K. Mai, Makromol. Chem., 187, 1787 (1986).

4. M.-F. Cheung, A. Golovoy, H. K. Plummer, and H. Vanoene, Polymer, 31, 2299 (1990).

5. M.-F. Cheung, A. Golovoy, and H. Vanoene, Polymer, 31, 2307 (1990).

6. M.-F. Cheung and H. K. Plummer, Polym. Bull., 26, 349 (1991).

7. S. Akhtar and J. L. White, Polym. Eng. Sci., 31, 84 (1991).

8. Q. Guo, J. Huang, and T. Chen, Polym. Bull., 20, 517 (1988).

9. Q. Guo, J. Huang, T. Chen, H. Zhang, Y. Yang, C. Hou, and Z. Feng, Polym. Eng. Sci., 30, 44 (1990).

10. X. Zhang and Y. Wang, Polymer, 30, 1867 (1989).

11. C. K. Sham, C. H. Lau, D. J. Williams, F. E. Karasz, and W. J. MacKnight, Br. Polym. J., 20, 149 (1988).

12. Z. Wu, Y. Zheng, X. Yu, T. Nakamura, and R. Yosomiya, Angew. Makromol. Chem., 171, 119 (1989).

13. Z. Wu, Y. Zheng, H. Yan, T. Nakamura, T. Nozawa, and R. Yosomiya, Angew. Makromol. Chem., 173, 163 (1989).

14. S. Nakata, M. Kakimoto, and Y. Imai, Polym. J., 20, 80 (1990).

15. H. Hayashi, S. Nakata, M. Kakimoto, and Y. Imai, J. Appl. Polym. Sci., 49, 1241 (1993).

16. M. Matsuura, H. Saito, S. Nakata, Y. Imai, and T. Inoue, Polymer, 33, 3210 (1992).

17. S. Nakata, M. Kakimoto, and Y. Imai, Polymer, 33, 3873 (1992).

18. P. W. Morgan, Macromolecules, 10, 1381 (1977). 